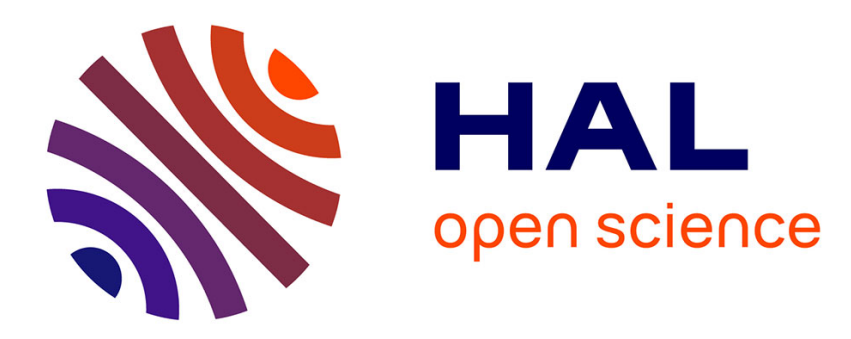

\title{
Théâtre et danse de l'Inde du Sud : deux publications récentes
}

\author{
Christine Guillebaud
}

\section{To cite this version:}

Christine Guillebaud. Théâtre et danse de l'Inde du Sud: deux publications récentes. Cahiers d'ethnomusicologie, 2000, 13, pp.251-253. hal-01891136

\section{HAL Id: hal-01891136 https://hal.science/hal-01891136}

Submitted on 9 Oct 2018

HAL is a multi-disciplinary open access archive for the deposit and dissemination of scientific research documents, whether they are published or not. The documents may come from teaching and research institutions in France or abroad, or from public or private research centers.
L'archive ouverte pluridisciplinaire HAL, est destinée au dépôt et à la diffusion de documents scientifiques de niveau recherche, publiés ou non, émanant des établissements d'enseignement et de recherche français ou étrangers, des laboratoires publics ou privés. 


\section{Théâtre et danse de l'Inde du Sud: deux publications récentes}

Inde du Sud. Kutiyattam. Théâtre classique des temples du Kerala. Troupe de Kutiyattam du Kerala Kalamandalam (direction musicale: P.K. Nambiar), Enregistrements: Roger Filipuzzi (Centre Mandapa); coordination des textes (français, anglais, allemand): Milena Salvini. 1 CD Ocora Radio France C 560143, 1999. Durée: $75^{\prime} 00$.

Enregistré à Paris en février 1998, ce disque présente un extrait tiré de l'Abhisheka Natakam (Bhasa, III siècle) intitulé Balivadham (relatant la mort de Bali), tel qu'il a été représenté sur scène par la troupe du Kerala Kalamandalam. Deux publications antérieures avaient déjà présenté des enregistrements de Kathakali ${ }^{\prime}$, autre forme théâtrale originaire de l'Etat du Kerala. A la différence de ce dernier où le texte du poète est confié à deux chanteurs, le Kutiyattam met l'acteur au centre de son dispositif théâtral puisque celui-ci assure à la fois la récitation vocale du texte, sa traduction littérale en mudra (langage codifié des mains), sa mise en expression corporelle (yeux, visage, postures, déplacements) ainsi que son "commentaire» (extrapolations improvisées). Les interventions musicales sont assurées par des instrumentistes situés en fond de scène (tambours mizhavu et edakka, cymbales kuzhitalam et parfois hautbois kurumkuzhal).

Les enregistrements présentés sont étonnants par leur réalisme restituant fidèlement la structure spatiale de la scène. C'est en effet dans la représentation théâtrale que se situe sans aucun doute le contexte de cette musique. Totalement imbriquée dans le jeu théâtral, elle intervient sous des formes multiples, venant par exemple ponctuer la récitation du texte, fournir le cadre rythmique de référence pour la gestuelle de l'acteur, souligner certains détails du récit par des interventions de type «bruitages", rendre compte de la dynamique des actions par des changements d'intensité et de tempo... La qualité de la prise de son permet à l'auditeur de se représenter les différents niveaux de la scène sans lesquels l'ensemble des aspects sonores de ce théâtre (récitation du texte, cris, musique) n'auraient aucune raison d'être. De même, la transcription du texte sanskrit ainsi que sa traduction, données dans le livret d'accompagnement, permettent à l'auditeur de replacer ces multiples interventions sonores dans l'intrigue du récit. Le fait que les enregistrements aient été réalisés au cours d'une représentation en France, c'està-dire en dehors de leur contexte culturel d'origine, n'interdit pas leur écoute et leur appréciation puisque le contexte scénique y est restitué de la même façon qu'au Kerala. Cependant, l'auteur aurait dû clairement spécifier les conditions générales de la performance, certes difficilement transposables sur une scène parisienne, mais qui, nous pensons, ont dû nécessiter des "aménagements» de la part des praticiens: durée de la représentation (d'une nuit entière à parfois plusieurs nuits), public de lettrés maîtrisant le sanskrit et le langage des mudra, espace de jeu situé dans l'enceinte même du temple...

Musiques, chants et rythmes du Kathakali. Le Mahâbhârata. 1 CD Auvidis/Ethnic B 6778 (1993); Musiques, chants et rythmes du Kathakali. Le Ramâyâna. 1 CD Auvidis/Ethnic B 6779 (1993). 
Présenter le Kutiyattam par le biais du seul support audio est sans aucun doute un exercice difficile puisque le jeu gestuel et vocal de l'acteur ainsi que la musique sont intimement liés. Nous attendions néanmoins de la part de l'auteur une présentation plus rigoureuse et plus approfondie des règles générales de récitation du texte sanskrit ainsi que de celles régissant le système musical. En d'autres termes, là où nous pensions trouver des clés pour l'écoute, nous n'avons que des listes de termes sanskrit non référencés, retranscrits parfois avec des erreurs et accompagnés d'explications souvent confuses. Concernant le jeu vocal de l'acteur (vachikabhinaya), certaines imprécisions (imputables peut-être à des problèmes de traduction) méritent d'être reprises ici. Tout d'abord, le texte poétique est bien récité et non "chanté»" par l'acteur (p. 1, p. 5); les termes sont d'ailleurs clairement distincts en sanskrit (pathya désignant la récitation, gita désignant le chant). Ensuite, le terme svara désigne dans la théorie classique les hauteurs musicales et non des "modes musicaux" (p. 5); erreur qui rend la présentation d'autant plus confuse que l'auteur ajoute: «[la manière de 'chanter' les svara] [...] diffère de la manière d'interpréter un raga en concert classique $[. .$.$] bien que les svara du Kutiyattam soient quelquefois appelés$ aussi raga ". L'auteur a recueilli auprès d'un praticien de ce théâtre une liste de vingtet-un svara ainsi que les situations dramatiques types auxquelles ils seraient associés. Cette nomenclature reste peu explicite à l'écoute des enregistrements; il est clair que la récitation ne suit aucunement des modes musicaux, mais une organisation des hauteurs proche de la récitation védique (trois hauteurs udatta, anudatta et svarita), règles générales qui auraient pu être développées davantage dans la notice.

Concernant les aspects purement musicaux, nous aurions aimé trouver une description plus rigoureuse des cycles rythmiques (tala), notamment dans leur subdivision interne en cellules, au moyen de schémas où apparaitraient par exemple les temps forts et faibles du cycle. La construction interne des tala n'a pas toujours été détaillée; de plus le tala de huit temps se nomme chempata et non chempa (autre tala correspondant à un cycle de dix temps)... Enfin, aucune information n'est donnée sur les modalités collectives du jeu de l'orchestre, notamment sur la répartition des rôles entre les différents instrumentistes (cymbales marquant les temps forts, un ou deux tambours mizhavu assurant la même fonction, un mizhavu «soliste» introduisant des improvisations rythmiques, interventions irrégulières du tambour edakka...). Autant de clés qui auraient dirigé davantage l'auditeur dans l'écoute d'une musique peu connue et difficile à appréhender en dehors du contexte de la représentation.

Inde du Sud. Margam. L'Intégrale du Bharatanâtyam. Compositions de Vidwan Madurai Sri N.Krishnan. Enregistrements: Errol Mailbach, Laurent Aubert; texte (français, anglais): Manjula Lusti-Narasimhan, Genève. 2 CD Archives Internationales de Musique Populaire AIMP LXI-LXII, VDE-1004-1005, 2000. Durée: 78'11" (CD1) et 61'04" (CD 2).

Cette série d'enregistrements présente le répertoire de la musique de danse Bharatanâtyam originaire de l'Etat du Tamil Nadu. Organisée selon les règles de la tradition classique carnatique, cette musique à la fois vocale et instrumentale a la 
particularité d'être dansée, bien que ses interprètes soient par ailleurs des musiciens concertistes. Les pièces sont ici restituées selon la logique du margam, ensemble des différentes séquences constituant un récital complet de danse. Celles-ci s'ordonnent dans une certaine progression rythmique et mélodique, sollicitant les différents niveaux techniques et les registres expressifs de la danseuse. De la danse pure (nrtta) à la danse narrative (nrtya) impliquant chacune des techniques et des catégories expressives propres, la structure du spectacle allie savamment les multiples modalités du mouvement dansé: séquences de frappes rythmiques avec les pieds exprimées verbalement par des syllabes rythmiques (jati), parties narratives engageant les subtiles expressions du visage de la danseuse (abhinaya)... [Petite parenthèse linguistique à propos de du terme sanskrit abhinaya: traduit d'abord dans la notice par le verbe «éduquer» (p. 6), le terme est analysé plus loin (p. 16) avec une étymologie inversée. «naya» est en fait construit sur la racine NI-, "conduire», et ne signifie donc pas "devant». De même, "abhi» ne signifie pas "apporter», mais "vers»! Etymologiquement, le terme abhinaya désignerait donc le fait de «conduire» quelque chose "vers» une autre. La traduction retenue par l'auteur ("éduquer ») est-elle la plus appropriée en matière d'esthétique?...]

Dans le Bharatanâtyam, la structure des chorégraphies peut être totalement fixe (comme dans l'alarippu dansé au début du récital) ou intégrer des parties improvisées de complexité variable (cas du varnam). La musique et le texte chanté sont eux aussi déterminés à l'avance (notamment les raga et tala) puisqu'ils sont l'œuvre de compositeurs. Les pièces ici enregistrées sont des compositions de Vidwan Madurai Sri N. Krishnan, directeur de l'école du Sri Bharatalaya, et sont interprétées par des musiciens dont la plupart sont membres de cette institution. Professionnels de haut niveau, ils maitrisent aussi totalement l'exercice de l'enregistrement en studio, espace faisant aujourd'hui partie intégrante de la pratique des musiciens classiques indiens. Cependant, la performance y perd sans aucun doute en qualité. Une musique de danse enregistrée hors de la scène exclut par exemple le timbre des sonnailles de la danseuse (accrochées à ses chevilles), élément dynamique pourtant essentiel notamment dans les séquences purement rythmiques. De même, le discours musical se trouve totalement privé du jeu des interactions danseuse/musiciens alors qu'elles constituent le moteur des ajustements de tempo, de la dynamique du chant, de la profondeur expressive du texte... La prise de son studio, certes d'une toute autre qualité que celles réalisées en général en Inde, ne peut prétendre restituer la dynamique de la performance scénique (progression dynamique et expressive du début à la fin du récital, espace de la danseuse par rapport à celui de l'orchestre, interactions avec le public). Le résultat final nous a paru quelque peu désincarné. Plutôt dommage pour une publication présentée par une praticienne confirmée de Bharatanâtyam.

Christine Guillebaud 